Erratum

\title{
Sequence and expression characteristics of three G-box binding factor cDNAs from Brassica napus
}

\author{
Stephan Waldmüller and Gerhard Link* \\ University of Bochum, Plant Cell Physiology and Molecular Biology, Universitätsstrasse 150, D-44780 Bochum, \\ Germany ("author for correspondence)
}

Plant Molecular Biology 29: 507-517, 1995.

Typography of the title should be as printed above. 